\title{
Effect of Resonance on the Motion of Two Cylindrical Rigid Bodies
}

\author{
M. R. Hassan1, Baby Kumari², Md. Aminul Hassan³, Payal Singh², B. K. Sharma4 \\ ${ }^{1}$ P. G. Department of Mathematics, S. M. College, T. M. Bhagalpur University, Bhagalpur, India \\ ${ }^{2}$ Research Scholar, T. M. Bhagalpur University, Bhagalpur, India \\ ${ }^{3}$ GTE, Yeshwanthpur, Bangalore, India \\ ${ }^{4}$ Department of Mathematics, SBS College, University of Delhi, New Delhi, India \\ Email:hassansmc@gmail.com,mahassan012@gmail.com
}

How to cite this paper: Hassan, M.R., Kumari, B., Hassan, Md.A., Singh, P. and Sharma, B.K. (2016) Effect of Resonance on the Motion of Two Cylindrical Rigid Bodies. International Journal of Astronomy and Astrophysics, 6, 555-574.

http://dx.doi.org/10.4236/ijaa.2016.64040

Received: October 8, 2016

Accepted: December 27, 2016

Published: December 30, 2016

Copyright $\odot 2016$ by authors and Scientific Research Publishing Inc. This work is licensed under the Creative Commons Attribution International License (CC BY 4.0). http://creativecommons.org/licenses/by/4.0/

\begin{abstract}
The effect of resonance on the motion of two cylindrical rigid bodies has been studied in the light of Bhatnagar [1] [2] [3] and under some defined axiomatic restrictions. Here we have calculated variation in Eulerian angles due to resonance in terms of orbital elements and unperturbed Eulerian angles.
\end{abstract}

\section{Keywords}

Inertia Ellipsoid, Ellipsoids of Revolution, Symmetrical Bodies, Orientation of the Bodies, Principal Axes, Eulerian Angles, Critical Points, Perturbations, Averaging of Hamiltonian, Resonance

\section{Introduction}

Russel [4] studied the motion of two spherical rigid bodies. In same way, Kopal [5] extended the previous work of Russel [4]; Cowling [6], Sterne [7] and Brouwer [8] generalized the work of previous authors by considering the lean angle and eccentricity as the small quantities. Johnson and Kane [9] extended the work of above authors by imposing some axiomatic restrictions as follows:

1) The inertia ellipsoids of two rigid bodies $A$ and $B$ for their respective mass centre $A^{*}$ and $B^{*}$ are ellipsoids of revolution.

2) Either the distance between $A^{*}$ and $B^{*}$ is considerably greater than the greatest dimension of either body or the ellipticities of the inertia ellipsoids of $A$ and $B$ are small.

3) The angular velocities of $A$ and $B$ in an inertial frame of reference $R$ are in- 
itially parallel to the symmetrical axes of $A$ and $B$ respectively.

4) The mass centers $A^{*}$ and $B^{*}$ move in plane whose orientation is fixed in $R$.

Bhatnagar [3], Elipe and Miguel [10], Choudhary and Mishra [11], Mercedes and Elipe [12] have discussed the problem similar to the works of the author of early thirties and forties. But Milution Marjanov [13] has discussed the problem on the cause of resonant motions of celestial bodies in an inhomogeneous gravitational field. He has shown that, when eccentricities of the orbits differ from zero and cross section of the ellipsoids of inertia with orbital plane differs from the circle, the two-cycle resonance is the most stable one. Further Milution Marjanov [13] has discussed the effect of resonance on the problem of two real bodies. He has shown that there are 22 periodic functions and all the variables are coupled. Moreover he established that the stability of the orbit i.e. periodicity of the motion requires 231 resonances.

In our present work, we have proposed to extend the work of Bhatnagar et al. [1] [2] [3] by taking into account the effect of resonance and imposing some modified axiomatic restrictions as follows:

1) The inertia ellipsoids $A$ and $B$ for their mass centers $A^{*}$ and $B^{*}$ are considered as general ellipsoids only but not the ellipsoids of revolution.

2) The angular velocities of $A$ and $B$ are initially parallel to one of the principal axes, which is perpendicular to the orbital plane of $A$ and $B$.

3) Only the periodic terms are taken and other terms are neglected.

4) The two rigid bodies are symmetrical and cylindrical.

On taking axioms second and fourth under consideration $I_{A_{1}}=I_{A_{2}}$ for $A$ and $I_{B_{1}}=I_{B_{2}}$ for $B$, more critical points are found than that found by Bhatnagar and Gupta [1] [2].

\section{Equations of Motion}

Let $A^{*}\left(A_{1}, A_{2}, A_{3}\right)$ be the mass center of the body $A$ in the rotating frame of reference $R^{\prime}$ having a variable orientation in the fixed frame of reference $R$ which is shown in Figure 1. Let $X, Y, Z$ be fixed right handed mutually perpendicular axes in $R$. Let us suppose that $A_{1}, A_{2}, A_{3}$ are lines parallel to the principal axes of $A$ at $A^{*}$. We assume that $X Y$-plane is normal to the angular momentum of the system about the centre of mass. Let $r$ be the distance between $A^{*}$ and $B^{*}, \theta$ be the angle between $A^{*} B^{*}$ and $x$-axis. Let us assume that $\psi_{A}, \theta_{A}, \varphi_{A}$ be the Eulerian angle with the help of the principal axes $A_{1}, A_{2}, A_{3}$ of the body $A$ at its centre of mass $A^{*}$ oriented with the fixed axes $X, Y, Z$ respectively. Similarly $\psi_{B}, \theta_{B}, \varphi_{B}$ be the Eulerian angles with the help of the principal axes $B_{1}, B_{2}, B_{3}$ of the body $B$ at its centre of mass $B^{*}$, oriented with the fixed axes $X, Y, Z$ respectively.

Let $p_{1}, p_{2}, p_{3}, p_{4}, p_{5}, p_{6}, p_{7}, p_{8}$ be generalized momenta corresponding to the generalized co-ordinates $r, \theta, \psi_{A}, \theta_{A}, \varphi_{A}, \psi_{B}, \theta_{B}, \varphi_{B}$ respectively. Let $I_{A_{i}}$ and $I_{B_{i}}(i=1,2,3)$ be the principal moments of inertia, $\omega_{A_{i}}$ and $\omega_{B_{i}}(i=1,2,3)$ be the components of the angular velocities of body $A$ and $B$ respectively. If $m_{A}$ and $m_{B}$ be the masses of the two cylinders $A$ and $B$ respectively then the total kinetic energy of the system is given by 


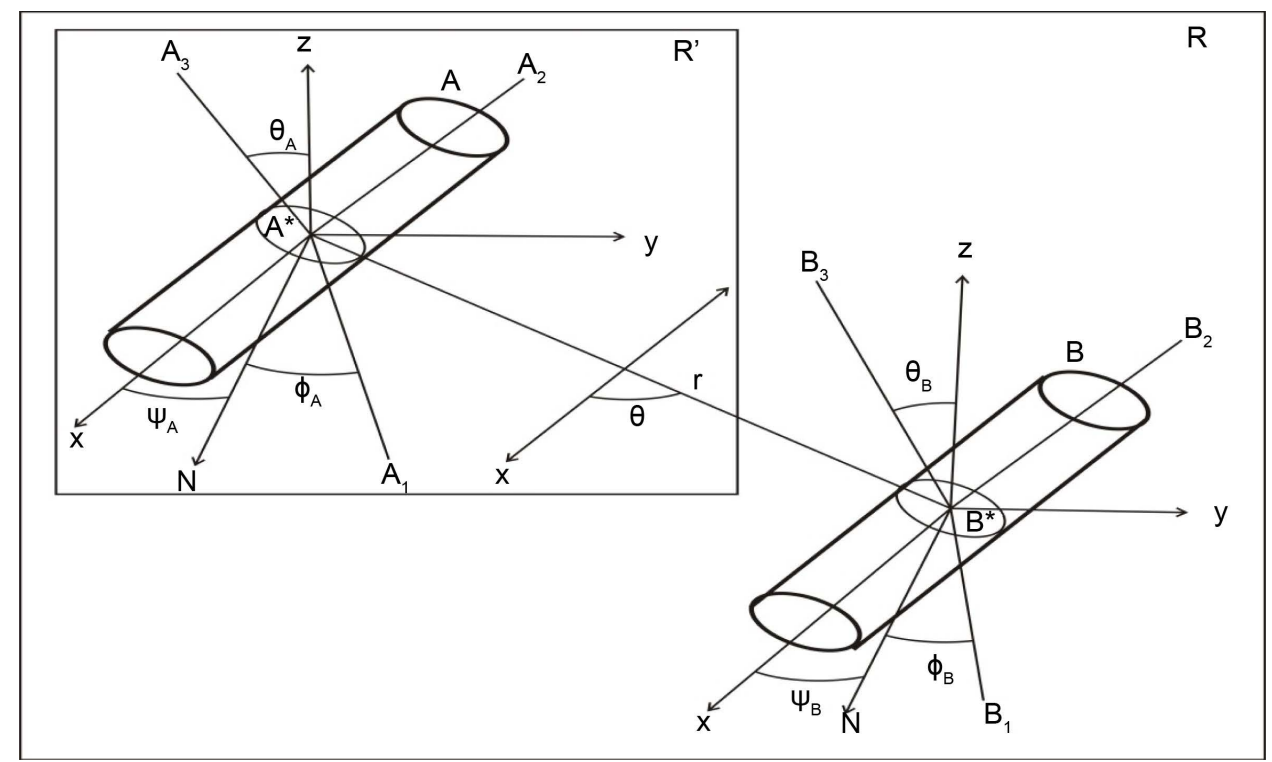

Figure 1. Orientation of the bodies.

$$
T=T_{\text {trans }}+T_{\text {rot }}=\frac{m_{A}+m_{B}}{m_{A} m_{B}}\left(T_{0}+T_{1}\right),
$$

where, $T_{0}=$ kinetic energy of $A$ and $B$ due to translation.

$$
=\frac{m_{A} m_{B}}{2\left(m_{A}+m_{B}\right)}\left(\dot{r}^{2}+r^{2} \dot{\theta}^{2}\right) .
$$

$T_{1}=$ Sum of kinetic energy of $A$ and $B$ due to rotation about the principle axes.

$$
=\frac{1}{2}\left[I_{A_{1}} \omega_{A_{1}}^{2}+I_{A_{2}} \omega_{A_{2}}^{2}+I_{A_{3}} \omega_{A_{3}}^{2}+I_{B_{1}} \omega_{B_{1}}^{2}+I_{B_{2}} \omega_{B_{2}}^{2}+I_{B_{3}} \omega_{B_{3}}^{2}\right] \text {. }
$$

If $\psi, \theta, \varphi$ be the Eulerian angles shown in Figure 1 then the components of angular velocity are given by

$$
\omega_{1}=\{\dot{\psi} \sin \theta \sin \varphi+\dot{\theta} \cos \varphi\}, \omega_{2}=\{\dot{\theta} \sin \varphi-\dot{\psi} \sin \theta \cos \varphi\}, \omega_{3}=\{\dot{\psi} \cos \theta+\dot{\varphi}\}
$$

Thus the combination of Equations (1), (2), (3) and (4) yields

$$
\begin{aligned}
T= & \frac{1}{2}\left(\dot{r}^{2}+r^{2} \dot{\theta}^{2}\right)+\frac{m_{A}+m_{B}}{2 m_{A} m_{B}}\left[I_{A_{1}}\left(\dot{\psi}_{A} \sin \theta_{A} \sin \phi_{A}+\dot{\theta}_{A} \cos \varphi_{A}\right)^{2}\right. \\
& +I_{A_{2}}\left(\dot{\psi}_{A} \sin \theta_{A} \cos \varphi_{A}-\dot{\theta}_{A} \sin \varphi_{A}\right)^{2}+I_{A_{3}}\left(\dot{\psi}_{A} \cos \theta_{A}+\dot{\varphi}_{A}\right)^{2} \\
& +I_{B_{1}}\left(\dot{\psi}_{B} \sin \theta_{B} \sin \varphi_{B}+\dot{\theta}_{B} \cos \varphi_{B}\right)^{2}+I_{B_{2}}\left(\dot{\psi}_{B} \sin \theta_{B} \cos \varphi_{B}-\dot{\theta}_{B} \sin \varphi_{B}\right)^{2} \\
& \left.+I_{B_{3}}\left(\dot{\psi}_{B} \cos \theta_{B}-\dot{\varphi}_{B}\right)^{2}\right] .
\end{aligned}
$$

Since for cylindrical bodies $I_{A_{1}}=I_{A_{2}}$ and $I_{B_{1}}=I_{B_{2}}$ hence from the Equation (5), we get

$$
\begin{aligned}
T= & \frac{1}{2}\left(\dot{r}^{2}+r^{2} \dot{\theta}^{2}\right)+\frac{m_{A}+m_{B}}{2 m_{A} m_{B}}\left[I_{A_{1}}\left(\dot{\psi}_{A}^{2} \sin ^{2} \theta_{A}+\dot{\theta}_{A}^{2}\right)+I_{A_{3}}\left(\dot{\psi}_{A} \cos \theta_{A}+\dot{\varphi}_{A}\right)^{2}\right. \\
& \left.+I_{B_{1}}\left(\dot{\psi}_{B}^{2} \sin ^{2} \theta_{B}+\dot{\theta}_{B}^{2}\right)+I_{B_{2}}\left(\dot{\psi}_{B} \cos \theta_{B}+\dot{\varphi}_{B}\right)^{2}\right] .
\end{aligned}
$$


The generalized momenta $p_{i}\{i=1,2, \cdots 8\}$ corresponding to generalized coordinates $q_{i}\{i=1,2, \cdots 8\}$ are given by the relations

$$
p_{i}=\frac{\partial T}{\partial \dot{q}_{i}},
$$

where, $q_{1}=r, q_{2}=\theta, q_{3}=\psi_{A}, q_{4}=\theta_{A}, q_{5}=\varphi_{A}, q_{6}=\psi_{B}, q_{7}=\theta_{B}, q_{8}=\varphi_{B}$,

i.e. $p_{1}=\dot{r}, p_{2}=r^{2} \dot{\theta}$,

$$
\begin{gathered}
p_{3}=\frac{m_{A}+m_{B}}{m_{A} m_{B}}\left[I_{A_{1}}\left(\dot{\psi}_{A} \sin ^{2} \theta_{A}\right)+I_{A_{3}}\left(\dot{\psi}_{A} \cos ^{2} \theta_{A}+\dot{\varphi}_{A} \cos \theta_{A}\right)\right], \\
\therefore \dot{\theta}_{A}=\frac{m_{A} m_{B}}{m_{A}+m_{B}} \times \frac{p_{4}}{I_{A_{1}}}, \\
p_{5}=\frac{m_{A}+m_{B}}{m_{A} m_{B}}\left[I_{A_{3}}\left(\dot{\psi}_{A} \cos \theta_{A}+\dot{\varphi}_{A}\right)\right], \quad p_{3}-p_{5} \cos \theta_{A}=\frac{m_{A}+m_{B}}{m_{A} m_{B}}\left[I_{A_{1}}\left(\dot{\psi}_{A} \sin ^{2} \theta_{A}\right)\right] . \\
p_{4}=\frac{m_{A}+m_{B}}{m_{A} m_{B}} I_{A_{1}} \dot{\theta}_{A} . \\
\therefore \dot{\psi}_{A}=\frac{m_{A} m_{B}}{m_{A}+m_{B}} \times \frac{1}{I_{A_{1}} \sin ^{2} \theta_{A}}\left(p_{3}-p_{5} \cos \theta_{A}\right) .
\end{gathered}
$$

From $p_{5}$, we get

$$
\begin{gathered}
\dot{\varphi}_{A}=\frac{m_{A} \cdot m_{B}}{m_{A}+m_{B}}\left[\frac{p_{5}}{I_{A_{3}}}-\frac{1}{I_{A_{1}} \sin ^{2} \theta_{A}}\left(p_{3}-p_{5} \cos \theta_{A}\right)\right] \\
p_{6}=\frac{\partial T}{\partial \dot{\psi}_{B}}=\frac{m_{A}+m_{B}}{m_{A} m_{B}}\left[I_{B_{1}}\left(\dot{\psi}_{B} \sin ^{2} \theta_{B}\right)+I_{B_{3}}\left(\dot{\psi}_{B} \cos ^{2} \theta_{B}+\dot{\varphi}_{B} \cos \theta_{B}\right)\right], \\
p_{7}=\frac{\partial T}{\partial \dot{\theta}_{B}}=\frac{m_{A}+m_{B}}{m_{A} m_{B}} I_{B_{1}} \dot{\theta}_{B} \\
\Rightarrow \dot{\theta}_{B}=\frac{m_{A} m_{B}}{m_{A}+m_{B}} \times \frac{p_{7}}{I_{B_{1}}} . \\
p_{8}=\frac{\partial T}{\partial \dot{\varphi}_{B}}=\frac{m_{A}+m_{B}}{m_{A} m_{B}}\left[I_{B_{3}}\left(\dot{\psi}_{B} \cos \theta+\dot{\varphi}_{B}\right)\right], \\
p_{6}-p_{8} \cos \theta=\frac{m_{A}+m_{B}}{m_{A} m_{B}}\left[I_{B_{3}} \dot{\psi}_{B} \sin ^{2} \theta_{B}\right] \\
\Rightarrow \dot{\psi}_{B}=\frac{m_{A} m_{B}}{m_{A}+m_{B}} \times \frac{1}{I_{B_{1}} \sin ^{2} \theta_{B}}\left[p_{6}-p_{8} \cos \theta_{B}\right] .
\end{gathered}
$$

From $p_{8}$, we get

$$
\dot{\varphi}_{B}=\frac{m_{A} m_{B}}{m_{A}+m_{B}}\left[\frac{p_{8}}{I_{B_{3}}}-\frac{1}{I_{B_{1}} \sin ^{2} \theta_{B}}\left(p_{6}-p_{8} \cos \theta_{B}\right)\right] .
$$

Introducing $\dot{q}_{i}$ in the Equation (6), we get

$$
\left.\begin{array}{rl}
T= & \frac{1}{2}\left(p_{1}^{2}+\frac{p_{2}^{2}}{r^{2}}\right)+\frac{1}{2}\left(\frac{m_{A} m_{B}}{m_{A}+m_{B}}\right)\left[\frac{1}{I_{A_{1}} \sin ^{2} \theta_{A}}\left\{\left(p_{3}-p_{5} \cos \theta_{A}\right)^{2}+p_{4}^{2} \sin ^{2} \theta_{A}\right\}\right) \\
& \left.+\frac{p_{5}^{2}}{I_{A_{3}}}+\frac{1}{I_{B_{1}} \sin ^{2} \theta_{B}}\left\{\left(p_{6}-p_{8} \cos \theta_{B}\right)^{2}+p_{7}^{2} \sin ^{2} \theta_{B}\right\}+\frac{p_{8}^{2}}{I_{B_{3}}}\right]
\end{array}\right\}
$$


Following Brouwer and Clemenc [14] the potential $V$ for the two bodies $A$ and $B$ is given by

$$
V=G \frac{m_{A}+m_{B}}{m_{A} m_{B}} \iint \frac{\mathrm{d} m_{A} \mathrm{~d} m_{B}}{r},
$$

where $r$ is the distance between two elements $\mathrm{d} m_{A}$ and $\mathrm{d} m_{B}$ of the two bodies $A$ and $B$ respectively and $G$ is the gravitational constant. The integration extends over total mass of two bodies.

From Equation (9), we get

$$
\left.\begin{array}{rl}
V= & \frac{\mu}{r}+\frac{\mu}{2 m_{A} r^{3}}\left[\left(I_{A_{3}}-I_{A_{1}}\right)\left\{1-3 \sin ^{2} \theta_{A} \sin ^{2}\left(\theta-\psi_{A}\right)\right\}\right] \\
& +\frac{\mu}{2 m_{B} r^{3}}\left[\left(I_{B_{3}}-I_{B_{1}}\right)\left\{1-3 \sin ^{2} \theta_{B} \sin ^{2}\left(\theta-\psi_{B}\right)\right\}\right]
\end{array}\right\},
$$

where $\mu=G\left(m_{A}+m_{B}\right)$.

The Hamiltonian function is given by

$$
H=T-V=H_{0}+H_{1},
$$

where, $H_{0}=$ unperturbed Hamiltonian

$$
=\frac{1}{2}\left[p_{1}^{2}+\frac{p_{2}^{2}}{r^{2}}\right]-\frac{\mu}{r} .
$$

$H_{1}=$ Perturbed Hamiltonian,

$$
\begin{aligned}
= & \frac{1}{2}\left(\frac{m_{A} m_{B}}{m_{A}+m_{B}}\right)\left[\frac{1}{I_{A_{1}} \operatorname{Sin}^{2} \theta_{A}}\left\{\left(p_{3}-p_{5} \cos \theta_{A}\right)^{2}+p_{4}^{2} \sin ^{2} \theta_{A}\right\}+\frac{p_{5}^{2}}{I_{A_{3}}}\right. \\
& \left.+\frac{1}{I_{B_{1}} \operatorname{Sin}^{2} \theta_{B}}\left\{\left(p_{6}-p_{8} \cos \theta_{B}\right)^{2}+p_{7}^{2} \sin ^{2} \theta_{B}\right\}+\frac{p_{8}^{2}}{I_{B_{3}}}\right] \\
& -\frac{\mu}{2 m_{A} r^{3}}\left[\left(I_{A_{3}}-I_{A_{1}}\right)\left\{1-3 \operatorname{Sin}^{2} \theta_{A} \sin ^{2}\left(\theta-\psi_{A}\right)\right\}\right] \\
& -\frac{\mu}{2 m_{B} r^{3}}\left[\left(I_{B_{3}}-I_{B_{1}}\right)\left\{1-3 \operatorname{Sin}^{2} \theta_{B} \sin ^{2}\left(\theta-\psi_{B}\right)\right\} .\right.
\end{aligned}
$$

The Canonical equations of motion are given by

$$
\begin{aligned}
& \dot{r}=\frac{\partial H}{\partial p_{1}}, \dot{\theta}=\frac{\partial H}{\partial p_{2}}, \dot{\psi}_{A}=\frac{\partial H}{\partial p_{3}}, \dot{\theta}_{A}=\frac{\partial H}{\partial p_{4}}, \dot{\varphi}_{A}=\frac{\partial H}{\partial p_{5}}, \\
& \dot{\psi}_{B}=\frac{\partial H}{\partial p_{6}}, \dot{\theta}_{B}=\frac{\partial H}{\partial p_{7}}, \dot{\varphi}_{B}=\frac{\partial H}{\partial p_{8}}, \\
& \dot{p}_{1}=-\frac{\partial H}{\partial r}, \dot{p}_{2}=-\frac{\partial H}{\partial \theta}, \dot{p}_{3}=-\frac{\partial H}{\partial \psi_{A}}, \dot{p}_{4}=-\frac{\partial H}{\partial \theta_{A}}, \\
& \dot{p}_{5}=-\frac{\partial H}{\partial \phi_{A}}, \dot{p}_{6}=-\frac{\partial H}{\partial \psi_{B}}, \dot{p}_{7}=-\frac{\partial H}{\partial \theta_{B}}, \dot{p}_{8}=-\frac{\partial H}{\partial \phi_{B}} .
\end{aligned}
$$

\section{Unperturbed Solutions}

The Hamilton-Jacobi Equation for the Hamiltonian $H_{0}$ is given by 


$$
\frac{\partial s}{\partial r}+H_{0}\left(t, r, \theta, \frac{\partial s}{\partial r}, \frac{\partial s}{\partial \theta}\right)=0
$$

The solution of the above equation is given by

$$
s=-\alpha_{1} t+\alpha_{2} \theta+\int_{r_{1}}^{r_{2}} \sqrt{2\left(\frac{\mu}{r}+\alpha_{1}\right)-\frac{\alpha_{2}^{2}}{r^{2}}} \mathrm{~d} r .
$$

Hence the solution of the problem can be given in term of the Keplerian elements $a, e, \omega, \tau$ as

$$
\left.\begin{array}{l}
\alpha_{1}=\frac{-\mu}{2 a}, \alpha_{2}=\left[\mu a\left(1-e^{2}\right)\right]^{1 / 2}, r^{2} \frac{\mathrm{d} \theta}{\mathrm{d} t}=\left[\mu a\left(1-e^{2}\right)\right]^{1 / 2}, \beta_{1}=-\tau, \beta_{2}=\omega \\
r=\frac{a\left(1-e^{2}\right)}{1-e \cos (\theta-\omega)}=a(1-e \cos E), \theta=\omega+\cos ^{-1}\left[\frac{(\cos E-e)}{(1-e \cos E)}\right] \\
E-e \sin E=n(t-\tau), n=\left(\frac{\mu}{a^{3}}\right)^{1 / 2}
\end{array}\right\}
$$

Here a,e, $\omega, t$ are the usual Keplerian elements, $E$ is the eccentric anomaly, $\alpha_{1}$ and $\alpha_{2}$ are constants of integration, $\beta_{1}$ and $\beta_{2}$ are generalized momenta variables corresponding to $\alpha_{1}$ and $\alpha_{2}$ respectively.

\section{Approximate Variational Equations Corresponding to Perturbed Hamiltonian}

The set of approximate variational equations may be given by averaging the Hamiltonian $H_{1}$. The averaged value of the Hamiltonian $H_{1}$ is given by

$$
\bar{H}_{1}=\frac{n}{2 \pi} \int_{0}^{2 \pi / n} H_{1} \mathrm{~d} t
$$

where $H_{1}$ is given by the Equation (12).

Here, we observe that by averaging the Hamiltonian, short-periodic terms are eliminated from the Hamilton-Jacobi equation. An approximate set of variational equations are given by

$$
\left.\dot{\alpha}_{1}=\frac{\partial \bar{H}_{1}}{\partial \beta_{1}}=0, \quad \dot{\alpha}_{2}=\frac{\partial \bar{H}_{1}}{\partial \beta_{2}}=0, \quad \dot{\beta}_{1}=\frac{\partial \bar{H}_{1}}{\partial \alpha_{1}}, \quad \dot{\beta}_{2}=\frac{\partial \bar{H}_{1}}{\partial \alpha_{2}}\right\}
$$

From the above equations, we get

$$
\alpha_{i}=\text { const }=\alpha_{0} \text { (say) }(i=1,2) .
$$

From Equation (14), we have

$$
\begin{aligned}
& a=-\frac{\mu}{2 \alpha_{1}}=-\frac{\mu}{2 \alpha_{0}}=\text { const. }=a_{0}, \\
& \mathrm{e}=\sqrt{1-\frac{\alpha_{2}^{2}}{\mu a}}=\text { const. }=\mathrm{e}_{0} .
\end{aligned}
$$

Also, 


$$
\begin{aligned}
& \dot{\beta}_{1}=\frac{\partial \bar{H}}{\partial \alpha_{1}}=\frac{3}{a^{2}\left(1-\mathrm{e}^{2}\right)^{3 / 2}}\left[-\frac{\left(I_{A_{3}}-I_{A_{1}}\right)}{2 m_{A}}\left(1-\frac{3}{2} \sin ^{2} \theta_{A}\right)-\frac{\left(I_{B_{3}}-I_{B_{1}}\right)}{2 m_{B}}\left(1-\frac{3}{2} \sin ^{2} \theta_{B}\right)\right], \\
& \dot{\beta}_{2}=\frac{\partial \bar{H}}{\partial \alpha_{2}}=\frac{3 n}{a^{2}\left(1-\mathrm{e}^{2}\right)^{2}}\left[-\frac{\left(I_{A_{3}}-I_{A_{1}}\right)}{2 m_{A}}\left(1-\frac{3}{2} \sin ^{2} \theta_{A}\right)-\frac{\left(I_{B_{3}}-I_{B_{1}}\right)}{2 m_{B}}\left(1-\frac{3}{2} \sin ^{2} \theta_{B}\right)\right] .
\end{aligned}
$$

For solving the Equations (17) and (18), we should know $\psi_{A}, \theta_{A}, \varphi_{A}, \psi_{B}, \theta_{B}, \varphi_{B}$ as function of time.

\section{Solutions for Generalized Co-Ordinates}

$\psi_{A}, \theta_{A}, \varphi_{A}, \psi_{B}, \theta_{B}, \phi_{B}, \tau$ and $\omega$ are generalized co-ordinates.

For the solution, we will use the Lagrange's equation of motion

$$
\frac{\mathrm{d}}{\mathrm{d} t}\left(\frac{\partial T}{\partial \dot{q}}\right)-\frac{\partial T}{\partial q}=\frac{-\partial V}{\partial q}
$$

where $T=$ kinetic energy and $V=$ Potential energy of the system given by the Equations (8) and (10) respectively.

From Equation (6), we get

$$
\begin{aligned}
T= & \frac{1}{2}\left(\dot{r}^{2}+r^{2} \dot{\theta}^{2}\right)+\frac{\left(m_{A}+m_{B}\right)}{2 m_{A} m_{B}}\left[I_{A_{1}}\left\{\dot{\psi}_{A}^{2} \sin ^{2} \theta_{A}+\dot{\theta}_{A}^{2}\right\}+I_{A_{3}}\left(\dot{\psi}_{A} \cos \theta_{A}+\dot{\varphi}_{A}\right)^{2}\right. \\
& \left.+I_{B_{1}}\left\{\dot{\psi}_{B}^{2} \sin ^{2} \theta_{B}+\dot{\theta}_{B}^{2}\right\}+I_{B_{3}}\left(\dot{\psi}_{B} \cos \theta_{B}+\dot{\varphi}_{B}\right)^{2}\right] . \\
& \Rightarrow \frac{\partial T}{\partial \psi_{A}}=0,
\end{aligned}
$$

and

$$
\begin{gathered}
\frac{\partial T}{\partial \dot{\psi}_{A}}=\frac{m_{A}+m_{B}}{2 m_{A} m_{B}}\left[I_{A_{1}}\left(2 \dot{\psi}_{A} \sin ^{2} \theta_{A}\right)+2 I_{A_{3}}\left(\dot{\psi}_{A} \cos \theta_{A}+\dot{\varphi}_{A}\right) \cos \theta_{A}\right] \\
=\frac{m_{A}+m_{B}}{m_{A} m_{B}}\left[I_{A_{1}} \dot{\psi}_{A} \sin ^{2} \theta_{A}+I_{A_{3}}\left(\dot{\psi}_{A} \cos ^{2} \theta_{A}+\dot{\varphi}_{A} \cos \theta_{A}\right)\right] . \\
\frac{\mathrm{d}}{\mathrm{d} t}\left(\frac{\partial T}{\partial \dot{\psi}_{A}}\right)=\frac{m_{A}+m_{B}}{m_{A} m_{B}}\left[\ddot{\psi}_{A}\left\{I_{A_{1}} \sin ^{2} \theta_{A}+I_{A_{3}} \cos ^{2} \theta_{A}\right\}+\ddot{\varphi}_{A} I_{A_{3}} \cos \theta_{A}\right. \\
\left.+\dot{\psi}_{A} \dot{\theta}_{A} \sin 2 \theta_{A}\left(I_{A_{1}}-I_{A_{3}}\right)-\dot{\varphi}_{A} \dot{\theta}_{A} I_{A_{3}} \sin \theta_{A}\right] .
\end{gathered}
$$

From Equation (12), we have

$$
\begin{aligned}
V= & \frac{\mu}{r}+\frac{\mu}{2 m_{A} r^{3}}\left[\left(I_{A_{3}}-I_{A_{1}}\right)\left\{1-3 \sin ^{2} \theta_{A} \sin ^{2}\left(\theta-\psi_{A}\right)\right\}\right] \\
& +\frac{\mu}{2 m_{B} r^{3}}\left[\left(I_{B_{3}}-I_{B_{1}}\right)\left\{1-3 \sin ^{2} \theta_{B} \sin ^{2}\left(\theta-\psi_{B}\right)\right\}\right] \\
\Rightarrow & \frac{\partial V}{\partial \psi_{A}}=\frac{3}{2} \frac{\mu}{m_{A} r^{3}}\left[\left(I_{A_{3}}-I_{A_{1}}\right) \sin ^{2} \theta_{A} \sin 2\left(\theta-\psi_{A}\right)\right] .
\end{aligned}
$$

For $q=\psi_{A}$.

The combination of Equations (19), (20), (21), (22) and (23) gives 


$$
\begin{aligned}
& \frac{m_{A}+m_{B}}{m_{A} m_{B}}\left[\ddot{\psi}_{A}\left\{I_{A_{1}} \sin ^{2} \theta_{A}+I_{A_{3}} \cos ^{2} \theta_{A}\right\}+\ddot{\varphi}_{A} I_{A_{3}} \cos \theta_{A}\right. \\
& \left.+\dot{\psi}_{A} \dot{\theta}_{A}\left(I_{A_{1}}-I_{A_{3}}\right) \sin 2 \theta_{A}-\dot{\varphi}_{A} \dot{\theta}_{A} I_{A_{3}} \sin \theta_{A}\right] \\
& =\frac{3 \mu_{A}}{2 m_{A} r^{3}}\left[\left(I_{A_{3}}-I_{A_{1}}\right) \sin ^{2} \theta_{A} \sin 2\left(\theta-\psi_{A_{0}}\right)\right] \\
& \Rightarrow \ddot{\psi}_{A}\left\{I_{A_{1}} \sin ^{2} \theta_{A}+I_{A_{3}} \cos ^{2} \theta_{A}\right\}+\ddot{\varphi}_{A} I_{A_{3}} \cos \theta_{A}+\dot{\psi}_{A} \dot{\theta}_{A}\left(I_{A_{1}}-I_{A_{3}}\right) \sin 2 \theta_{A}-\dot{\varphi}_{A} \dot{\theta}_{A} I_{A_{3}} \sin \theta_{A} \\
& =\frac{3 \mu \mu_{A}}{2 r^{3}}\left[\left(I_{A_{3}}-I_{A_{1}}\right) \sin ^{2} \theta_{A} \sin 2\left(\theta-\psi_{A}\right)\right] .
\end{aligned}
$$

This is the required Lagrange's equation of motion in $\psi_{A}$.

Again,

$$
\begin{gathered}
\frac{\partial T}{\partial \dot{\theta}_{A}}=\frac{m_{A}+m_{B}}{m_{A} m_{B}} I_{A_{1}} \dot{\theta}_{A}, \quad \frac{\mathrm{d}}{\mathrm{d} t}\left(\frac{\partial T}{\partial \dot{\theta}_{A}}\right)=\frac{m_{A}+m_{B}}{m_{A} m_{B}} I_{A_{1}} \ddot{\theta}_{A}, \\
\frac{\partial T}{\partial \theta_{A}}=\frac{m_{A}+m_{B}}{m_{A} m_{B}}\left\{\dot{\psi}_{A}^{2} \frac{\left(I_{A_{1}}-I_{A_{3}}\right)}{2} \sin 2 \theta_{A}-I_{A_{3}} \dot{\varphi}_{A} \dot{\psi}_{A} \sin \theta_{A}\right\}, \\
\frac{\partial V}{\partial \theta_{A}}=\frac{-3 \mu}{2 m_{A} r^{3}}\left[\left(I_{A_{3}}-I_{A_{1}}\right) \sin 2 \theta_{A} \sin ^{2}\left(\theta-\psi_{A}\right)\right] .
\end{gathered}
$$

Thus the Lagrange's equation of motion in $\theta_{A}$ is

$$
\begin{aligned}
& \frac{\mathrm{d}}{\mathrm{d} t}\left(\frac{\partial T}{\partial \dot{\theta}_{A}}\right)-\frac{\partial T}{\partial \theta_{A}}=\frac{-\partial V}{\partial \theta_{A}}, \\
& \text { i.e. } \frac{m_{A}+m_{B}}{m_{A} m_{B}}\left[I_{A_{1}} \ddot{\theta}_{A}-\dot{\psi}_{A}^{2} \frac{\left(I_{A_{1}}-I_{A_{3}}\right)}{2} \sin 2 \theta_{A}+\dot{\varphi}_{A} \dot{\psi}_{A} I_{A_{3}} \sin \theta_{A}\right] \\
& =\frac{3 \mu}{2 m_{A} r^{3}}\left[\left(I_{A_{3}}-I_{A_{1}}\right) \sin 2 \theta_{A} \sin ^{2}\left(\theta-\psi_{A}\right)\right] \\
& \ddot{\theta}_{A} I_{A_{1}}-\dot{\psi}_{A}^{2} \frac{\left(I_{A_{1}}-I_{A_{3}}\right)}{2} \sin 2 \theta_{A}+\dot{\varphi}_{A} \dot{\psi}_{A} I_{A_{3}} \sin \theta_{A} \\
& =\frac{3 \mu \mu_{A}}{2 r^{3}}\left[\left(I_{A_{3}}-I_{A_{1}}\right) \sin 2 \theta_{A} \sin ^{2}\left(\theta-\psi_{A}\right)\right] .
\end{aligned}
$$

Again,

$$
\begin{aligned}
& \frac{\partial T}{\partial \dot{\varphi}_{A}}=\frac{1}{2} \frac{\left(m_{A}+m_{B}\right)}{m_{A} m_{B}}\left[I_{A_{3}} 2\left(\dot{\psi} \cos \theta_{A}+\dot{\varphi}_{A}\right)\right], \\
& \frac{\partial T}{\partial \varphi_{A}}=0, \quad \frac{\partial V}{\partial \varphi_{A}}=0, \\
& \frac{\mathrm{d}}{\mathrm{d} t}\left(\frac{\partial T}{\partial \dot{\varphi}_{A}}\right)-\frac{\partial T}{\partial \varphi_{A}}=\frac{\partial V}{\partial \varphi_{A}} . \\
& \Rightarrow \frac{m_{A}+m_{B}}{m_{A} m_{B}}\left[\ddot{\psi}_{A} I_{A_{3}} \cos \theta_{A}+I_{A_{3}} \ddot{\varphi}_{A}-I_{A_{3}} \dot{\psi}_{A} \dot{\theta}_{A} \sin \theta_{A}\right]=0, \\
& \Rightarrow \ddot{\psi}_{A} I_{A_{3}} \cos \theta_{A}+\ddot{\varphi}_{A} I_{A_{3}}-\dot{\psi}_{A} \dot{\theta}_{A} I_{A_{3}} \sin \theta_{A}=0 .
\end{aligned}
$$

Similarly for $q=\psi_{B}, \theta_{B}, \varphi_{B}$. 


$$
\begin{aligned}
\ddot{\psi}_{B}\left\{I_{B_{1}} \sin ^{2} \theta_{B}+I_{B_{3}} \cos ^{2} \theta_{B}\right\}+\ddot{\varphi}_{B} I_{B_{3}} \cos \theta_{B}+\dot{\psi}_{B} \dot{\theta}_{B}\left(I_{B_{1}}-I_{B_{3}}\right) \sin 2 \theta_{B}-\dot{\varphi}_{B} \dot{\theta}_{B} I_{B_{3}} \sin \theta_{B} \\
=\frac{3 \mu \mu_{B}}{2 r^{3}}\left[\left(I_{B_{3}}-I_{B_{1}}\right) \sin ^{2} \theta_{B} \sin ^{2}\left(\theta-\psi_{B}\right)\right] . \\
\ddot{\theta}_{B} I_{B_{1}}-\dot{\psi}_{B}^{2}\left(\frac{I_{B_{3}}-I_{B_{1}}}{2}\right) \sin 2 \theta_{B}+\dot{\varphi}_{B} \dot{\psi}_{B} I_{B_{3}} \sin \theta_{B} \\
=\frac{3 \mu \mu_{B}}{2 r^{3}}\left[\left(I_{B_{3}}-I_{B_{1}}\right) \sin 2 \theta_{B} \sin ^{2}\left(\theta-\psi_{B}\right)\right], \\
\ddot{\psi}_{B} I_{B_{3}} \cos \theta_{B}+\dot{\varphi}_{B} I_{B_{3}}-\dot{\psi}_{B} \theta_{B} I_{B_{3}} \sin \theta_{B}=0 .
\end{aligned}
$$

We have assumed that the angular velocities $\omega_{A}$ and $\omega_{B}$ of bodies $A$ and $B$ are initially parallel to one of the principal axes which is perpendicular to the orbital plane. If we further assume that no torque (unperturbed motion) is acting on either of the two bodies then both the bodies will spin at a constant rate about that axes and the orientation with the axes will be fixed.

In terms of the Eulerian angles, we have

$$
\begin{aligned}
& \psi_{A}=\text { constant }=\psi_{A_{0}}, \quad \psi_{B}=\text { constant }=\psi_{B_{0}}, \\
& \theta_{A}=\text { constant }=\theta_{A_{0}}, \theta_{B}=\text { constant }=\theta_{B_{0}}, \\
& \dot{\varphi}_{A}=\text { constant }=\omega_{A}, \quad \dot{\varphi}_{B}=\text { constant }=\omega_{B}, \\
& \varphi_{A}=\omega_{A} t+\varphi_{A_{0}}, \varphi_{B}=\omega_{B} t+\varphi_{B_{0}} .
\end{aligned}
$$

In the case of perturbed motion, let us suppose that

$$
\left.\begin{array}{lll}
\psi_{A}=\psi_{A_{0}}+\eta_{A}, & \theta_{A}=\theta_{A_{0}}+\xi_{A}, & \varphi_{A}=w_{A} t+\varphi_{A_{0}}+\zeta_{A} \\
\psi_{B}=\psi_{B_{0}}+\eta_{B}, & \theta_{B}=\theta_{B_{0}}+\xi_{B}, & \varphi_{B}=w_{B} t+\varphi_{B_{0}}+\xi_{B}
\end{array}\right\}
$$

where $\theta_{A_{0}}, \psi_{A_{0}}, \varphi_{A_{0}}, \theta_{B_{0}}, \psi_{B_{0}}, \varphi_{B_{0}}$ are the constants corresponding to the torque-free solutions and $\xi_{A}, \xi_{B}, \eta_{A}, \eta_{B}, \zeta_{A}, \zeta_{B}$ are small quantities which are functions of time.

Since bodies are cylinders hence

$$
I_{A_{1}}=I_{A_{2}}=m_{A} \frac{3 a^{2}+4 l^{2}}{12}, I_{A_{3}}=\frac{m_{A} a^{2}}{2}, I_{B_{1}}=I_{B_{2}}=m_{B} \frac{3 a^{\prime 2}+4 l^{\prime 2}}{12}, I_{B_{3}}=\frac{m_{B} a^{\prime 2}}{2},
$$

where, $a=\operatorname{radius}$ of body $A, l=$ length of body $A, a^{\prime}=\operatorname{radius}$ of body $B, l^{\prime}=$ length of body $B$.

We replace $\varphi_{A}$ and $\varphi_{B}$ by their steady state value $\varphi_{A_{0}}$ and $\varphi_{B_{0}}$ respectively and using the Equation (30) and (31) in Equations (24), (25) and (26) and neglecting higher order terms, then from Equation (24), we have

$$
\left.\begin{array}{rl}
\ddot{\eta}_{A}-\frac{\omega_{A} I_{A_{3}}}{I_{A_{1}} \sin \theta_{A_{0}}} \dot{\xi}_{A}= & \frac{3 \mu \mu_{A}}{2\left(1-\mathrm{e}^{2}\right)^{3} a^{3} m_{A} I_{A_{1}} \sin \theta_{A_{0}}} \\
& \times\left[\left\{\left(I_{A_{3}}-I_{A_{1}}\right) \sin ^{2} \varphi_{A_{0}} \sin 2\left(\theta-\psi_{A_{0}}\right)\right\}\{1+\mathrm{e} \cos (\theta-\omega)\}^{3}\right] .
\end{array}\right\}
$$

From Equation (25), we have

$$
\left.\begin{array}{rl}
\ddot{\xi}_{A}+\frac{\omega_{A} I_{A_{3}} \sin \theta_{A_{0}}}{I_{A_{1}}} \dot{\eta}_{A}= & \frac{3 \mu \mu_{A}}{2 a^{3}\left(1-\mathrm{e}^{2}\right)^{3} m_{A} I_{A_{1}}} \\
& \times\left[\left\{\left(I_{A_{3}}-I_{A_{1}}\right) \sin 2 \theta_{A_{0}} \sin ^{2}\left(\theta-\psi_{A_{0}}\right)\right\}\{1+\mathrm{e} \cos (\theta-\omega)\}^{3}\right] .
\end{array}\right\}
$$


From Equation (26), we have

$$
\ddot{\eta}_{A} \cos \theta_{A_{0}}+\ddot{\zeta}_{A}=0 \text {. }
$$

Similarly for the body $B$ using Equations (30) and (31) in Equations (27), (28) and (29), we get

$$
\left.\begin{array}{rl}
\ddot{\eta}_{B}-\frac{\omega_{B} I_{B_{3}}}{I_{B_{1}} \sin \theta_{B_{0}}} \dot{\xi}_{B}= & \frac{3 \mu \mu_{B}}{2\left(1-\mathrm{e}^{2}\right)^{3} a^{3} m_{B} I_{B_{1}} \sin 2 \theta_{B_{0}}} \\
& \left.\times\left[\left(I_{B_{3}}-I_{B_{1}}\right) \sin ^{2} \varphi_{B_{0}} \sin 2\left(\theta-\psi_{B_{0}}\right)\right][1+\mathrm{e} \cos (\theta-\omega)]^{3},\right\} \\
\ddot{\xi}_{B}+\frac{I_{B_{3}} \omega_{B} \sin \theta_{B_{0}}}{I_{B_{1}}} \dot{\eta}_{B}= & \frac{3 \mu \mu_{B}}{2 a^{3}\left(1-\mathrm{e}^{2}\right)^{3} m_{B} I_{B_{1}}} \\
& \times\left[\left(I_{B_{1}}-I_{B_{3}}\right) \sin 2 \theta_{B} \sin ^{2}\left(\theta-\psi_{B_{0}}\right)\right][1+\mathrm{e} \cos (\theta-\omega)]^{3} .
\end{array}\right\}
$$

From Equation (29), we have

$$
\ddot{\eta}_{B} \cos \theta_{B_{0}}+\ddot{\varsigma}_{B}=0
$$

Integrating the Equation (36) and putting the value of $\eta_{B}$ in the Equation (37) and neglecting the secular terms, we get

$$
\left.\begin{array}{rl}
\ddot{\xi}_{A}+\eta_{A}^{2} \xi_{A}= & \mu_{A}\left[C_{1} \cos (\theta-\omega)+C_{2} \cos 2(\theta-\omega)+C_{3} \cos 3(\theta-\omega)\right. \\
& +C_{4} \cos 2\left(\theta-\psi_{A_{0}}\right)+C_{5} \cos \left(3 \theta-2 \psi_{A_{0}}-\omega\right) \\
& +C_{6} \cos \left(\theta-2 \psi_{A_{0}}+\omega\right)+C_{7} \cos \left(4 \theta-2 \psi_{A_{0}}-2 \omega\right) \\
& \left.+C_{8} \cos \left(5 \theta-2 \psi_{A_{0}}-3 \omega\right)+C_{9} \cos \left(\theta+2 \psi_{A_{0}}-3 \omega\right)\right]
\end{array}\right\}
$$

where $C_{1}, C_{2}, C_{3}, C_{4}, \cdots, C_{9}$ are constants independent of $t$ and $\eta_{A}=\frac{\omega_{A} I_{A_{3}}}{I_{A_{1}}}=n_{A}$.

Considering Kepler's equation up to the $1^{\text {st }}$ order approximation $\theta=n t$, the solution of the Equation (38) is given by

$$
\left.\left.\begin{array}{rl}
\xi_{A}= & X_{A} \cos l_{A}+\mu_{A}\left[\frac{C_{1} \cos (\theta-\omega)}{-n^{2}+n_{A}^{2}}+\frac{C_{2} \cos 2(\theta-\omega)}{-4 n^{2}+n_{A}^{2}}+\frac{C_{3} \cos 3(\theta-\omega)}{-9 n^{2}+n_{A}^{2}}\right. \\
& +\frac{C_{4} \cos 2\left(\theta-\psi_{A_{0}}\right)}{-4 n^{2}+n_{A}^{2}}+\frac{C_{5} \cos \left(3 \theta-2 \psi_{A_{0}}-\omega\right)}{-9 n^{2}+n_{A}^{2}}+\frac{C_{6} \cos \left(\theta-2 \psi_{A_{0}}+\omega\right)}{-n^{2}+n_{A}^{2}} \\
& +\frac{C_{7} \cos \left(4 \theta-2 \psi_{A_{0}}-\omega\right)}{-16 n^{2}+n_{A}^{2}}+\frac{C_{8} \cos \left(5 \theta-2 \psi_{A_{0}}-3 \omega\right)}{-25 n^{2}+n_{A}^{2}}+\frac{C_{9} \cos \left(\theta-2 \psi_{A_{0}}-3 \omega\right)}{-n^{2}+n_{A}^{2}}
\end{array}\right\}\right)
$$

Here we can see that if any one of the denominator vanishes, the motion is indeterminate at the point. It depends on the mean motion and the angular velocity of rotation of the body. There are many points at which resonance will occur but for discussion we have consider only one point $2 n=n_{A}$ and for other we can use the similar procedure. We further assume that $\left|2 n-n_{A}\right|$ is a small quantity and at the equilibrium point $2 n-n_{A}$ i.e. mean motion and angular velocity of the rigid body $A$ are in the ratio of 
$1: 2$. In order to study the behavior at this point we will follow the procedure of Brown and Shook [15].

\section{Resonance at the Critical Points}

From right hand side of Equation (39), we have $n_{A}=n, 2 n, 3 n, 4 n$ are the critical points. Here we consider $n_{A}=2 n$ for discussing resonance. Now we shall calculate the amplitude and period of vibration in the variable $\xi_{A}$.

We may write the Equation (39) as

$$
\ddot{\xi}_{A}+n_{A}^{2} \xi_{A}=\mu_{A} \frac{\partial H_{A}}{\partial \xi_{A}},
$$

where,

$$
\begin{aligned}
H_{A}= & \xi_{A}\left[C_{1} \cos (\theta-\omega)+C_{2} \cos 2(\theta-\omega)+C_{3} \cos (\theta-\omega)+C_{4} \cos 2\left(\theta-\psi_{A_{0}}\right)\right. \\
& +C_{5} \cos \left(3 \theta-2 \psi_{A_{0}}-\omega\right)+C_{6} \cos \left(\theta-2 \psi_{A_{0}}+\omega\right)+C_{7} \cos \left(4 \theta-2 \psi_{A_{0}}-2 \omega\right) \\
& \left.+C_{8} \cos \left(5 \theta-2 \psi_{A_{0}}-3 \omega\right)+C_{9} \cos \left(\theta+2 \psi_{A_{0}}-3 \omega\right)\right]
\end{aligned}
$$

The solution of the equation

$$
\ddot{\xi}_{A}+n_{A}^{2} \xi_{A}=0
$$

is periodic and given by

$$
\xi_{A}=X_{A} \cos l_{A}, l_{A}=n_{A} t+\xi_{A}, n_{A}=f\left(X_{A}\right) .
$$

Let $\xi_{A}$ be the function of two independent variable $X_{A}$ and $l_{A}$ i.e. $\xi_{A}=\xi_{A}\left(X_{A}, l_{A}\right)$. The Equation (41) may be written as

$$
\frac{\partial^{2} \xi_{A}}{\partial l_{A}^{2}}+\xi_{A}=0
$$

Then

$$
\frac{\mathrm{d} \xi_{A}}{\mathrm{~d} t}=\frac{\partial \xi_{A}}{\partial l_{A}} \cdot \frac{\mathrm{d} l_{A}}{\mathrm{~d} t}+\frac{\partial \xi_{A}}{\partial X_{A}} \cdot \frac{\mathrm{d} X_{A}}{\mathrm{~d} t} .
$$

We want to replace $\xi_{A}$ from Equation (40) by two new variables $X_{A}$ and $l_{A}$ which are related to $\xi_{A}$ by Equation (42). As we are replacing one variable by other two co-relations between the new variables is at our choice. Let us choose it in such a way that

$$
\frac{\partial \xi_{A}}{\partial l_{A}} \cdot \frac{\mathrm{d} l_{A}}{\mathrm{~d} t}+\frac{\partial \xi_{A}}{\partial X_{A}} \cdot \frac{\mathrm{d} X_{A}}{\mathrm{~d} t}=n_{A} \frac{\partial \xi_{A}}{\partial l_{A}}
$$

Using Equations (44) and (45), we get $\frac{\mathrm{d} \xi_{A}}{\mathrm{~d} t}=n_{A} \frac{\partial \xi_{A}}{\partial l_{A}}$.

As $l_{A}, X_{A}$ and $n_{A}$ are function of time $t$, therefore differentiating it with respect to $t$, we get

$$
\ddot{\xi}_{A}=\frac{\mathrm{d}^{2} \xi_{A}}{\mathrm{~d} t^{2}}=n_{A} \frac{\partial^{2} \xi_{A}}{\partial l_{A}^{2}} \cdot \frac{\mathrm{d} l_{A}}{\mathrm{~d} t}+\frac{\partial}{\partial X_{A}}\left(n_{A} \frac{\partial \xi_{A}}{\partial l_{A}}\right) \cdot \frac{\mathrm{d} X_{A}}{\mathrm{~d} t}
$$


Using Equations (40), (43) and (46), we get

$$
\eta_{A} \frac{\partial^{2} \xi_{A}}{\partial l_{A}^{2}}\left(\frac{\partial l_{A}}{d t}-n_{A}\right)+\frac{\partial}{\partial X_{A}}\left(n_{A} \frac{\partial \xi_{A}}{\partial l_{A}}\right) \cdot \frac{d X_{A}}{d t}=\mu_{A} \frac{\partial H_{A}}{\partial \xi_{A}} .
$$

Also from the Equation (46), we get

$$
\frac{\partial \xi_{A}}{\partial l_{A}}\left(\frac{\partial l_{A}}{\partial t}-n_{A}\right)+\frac{\partial \xi_{A}}{\partial X_{A}} \cdot \frac{\mathrm{d} X_{A}}{\mathrm{~d} t}=0
$$

Obviously the Equations (47) and (48) are linear equations in $\left(\frac{\mathrm{d} l_{A}}{\mathrm{~d} t}-n_{A}\right)$ and $\frac{\mathrm{d} X_{A}}{\mathrm{~d} t}$. So solving these equations for these variables, we get

$$
\begin{gathered}
\frac{\mathrm{d} X_{A}}{\mathrm{~d} t}=\frac{\mu_{A}}{K_{A}} \cdot \frac{\partial H_{A}}{\partial l_{A}} \\
\frac{\mathrm{d} l_{A}}{\mathrm{~d} t}=n_{A}-\frac{\mu_{A}}{K_{A}} \cdot \frac{\partial \xi_{A}}{\partial X_{A}} \cdot \frac{\partial H_{A}}{\partial \xi_{A}}=n_{A}-\frac{\mu_{A}}{K_{A}} \cdot \frac{\partial H_{A}}{\partial X_{A}}
\end{gathered}
$$

where, $K_{A}=\frac{\partial}{\partial X_{A}}\left(n_{A} \frac{\partial \xi_{A}}{\partial l_{A}}\right) \frac{\partial \xi_{A}}{\partial l_{A}}-n_{A} \frac{\partial^{2} \xi_{A}}{\partial l_{A}^{2}} \cdot \frac{\partial \xi_{A}}{\partial X_{A}}$ is a function of $X_{A}$ only.

Also,

$$
\begin{aligned}
H_{A}= & \frac{X_{A}}{2}\left[C_{1}\left\{\cos \left(\theta-\omega+l_{A}\right)+\cos \left(\theta-\omega-l_{A}\right)\right\}\right. \\
& \left.\left.+C_{2}\left\{\cos 2(\theta-\omega)+l_{A}\right)+\cos 2(\theta-\omega)-l_{A}\right)\right\} \\
& \left.\left.+C_{3}\left\{\cos 3(\theta-\omega)+l_{A}\right)+\cos 3(\theta-\omega)-l_{A}\right)\right\} \\
& +C_{4}\left\{\cos \left(\theta-\psi_{A_{0}}+l_{A}\right)+\cos \left(\theta-\psi_{A}-l_{A}\right)\right\} \\
& +C_{5}\left\{\cos \left(3 \theta-2 \psi_{A_{0}}-\omega+l_{A}\right)+\cos \left(3 \theta-2 \psi_{A_{0}}-\omega-l_{A}\right)\right\} \\
& +C_{6}\left\{\cos \left(\theta-2 \psi_{A_{0}}+\omega+l_{A}\right)+\cos \left(\theta-2 \psi_{A_{0}}+\omega-l_{A}\right)\right\} \\
& +C_{7}\left\{\cos \left(4 \theta-2 \psi_{A_{0}}-2 \omega+l_{A}\right)+\cos \left(4 \theta-2 \psi_{A_{0}}-2 \omega+l_{A}\right)\right\} \\
& +C_{8}\left\{\cos \left(5 \theta-2 \psi_{A_{0}}-3 \omega+l_{A}\right)+\cos \left(5 \theta-2 \psi_{A_{0}}-3 \omega-l_{A}\right)\right\} \\
& \left.+C_{9}\left\{\cos \left(\theta+2 \psi_{A_{0}}-3 \omega+l_{A}\right)+\cos \left(5 \theta-2 \psi_{A_{0}}-3 \omega-l_{A}\right)\right\}\right]
\end{aligned}
$$

As $n_{A}, K_{A}$ are function of $X_{A}$ only, we can write the Equation (51) into canonical form with new variables $\bar{X}_{A}$ and $R_{A}$ defined by

$$
\mathrm{d} \bar{X}_{A}=K_{A} \mathrm{~d} K_{A}, \quad \mathrm{~d} R_{A}=-n_{A} \mathrm{~d} \bar{X}_{A}=-n_{A} K_{A} \mathrm{~d} X_{A}
$$

As $\frac{\mathrm{d} \bar{X}_{A}}{\mathrm{~d} t}=\frac{\partial}{\partial l_{A}}\left(R_{A}+\mu_{A} H_{A}\right), \frac{\mathrm{d} l_{A}}{\mathrm{~d} t}=\frac{-\partial}{\partial X_{A}}\left(R_{A}+\mu_{A} H_{A}\right)$ so differentiating the Equation

(50) and putting the value of $\frac{\mathrm{d} X_{A}}{\mathrm{~d} t}$ and $\frac{\mathrm{d} l_{A}}{\mathrm{~d} t}$, we get

$$
\begin{aligned}
\frac{\mathrm{d}^{2} l_{A}}{\mathrm{~d} t^{2}}= & \frac{\mu_{A}}{K_{A}}\left[\frac{\partial n_{A}}{\partial X_{A}} \cdot \frac{\partial H_{A}}{\partial l_{A}}-n_{A} \frac{\partial^{2} H_{A}}{\partial l_{A} \partial X_{A}}-\frac{\partial^{2} H_{A}}{\partial l_{A} \partial X_{A}}\right] \\
& +\frac{\mu_{A}^{2}}{K_{A}^{2}}\left[\frac{\partial^{2} H_{A}}{\partial l_{A} \partial X_{A}} \cdot \frac{\partial H_{A}}{\partial X_{A}}-K_{A} \frac{\partial}{\partial X_{A}}\left(\frac{1}{K_{A}} \cdot \frac{\partial H_{A}}{\partial X_{A}}\right) \cdot \frac{\partial H_{A}}{\partial l_{A}}\right] .
\end{aligned}
$$


Neglecting higher powers of $\mu_{A}$, we get

$$
\frac{\mathrm{d}^{2} l}{\mathrm{~d} t^{2}}=\frac{\mu_{A}}{K_{A}}\left[\frac{\partial n_{A}}{\partial X_{A}} \cdot \frac{\partial H_{A}}{\partial l_{A}}-n_{A} \frac{\partial^{2} H_{A}}{\partial l_{A} \partial X_{A}}-\frac{\partial^{2} H_{A}}{\partial l_{A} \partial X_{A}}\right]
$$

Here we observe that $l_{A}$ and $l$ are present in $H_{A}$ only as the sum of the periodic terms with argument $\left\{i l_{A}-j(n t+\varepsilon)\right\}$ where $n$ and $\varepsilon$ are given constants, thus we have

$$
\frac{\partial H_{A}}{\partial t}=-\left(\frac{j n}{i}\right) \frac{\partial H_{A}}{\partial l_{A}}
$$

The Equation (53) can be written

$$
\frac{\mathrm{d}^{2} l}{\mathrm{~d} t^{2}}+\sum\left(i n_{\mathrm{A}}-j n\right)^{2} \frac{\mu_{\mathrm{A}}}{i K_{\mathrm{A}}}\left\{\frac{\partial}{\partial X_{A}}\left(\frac{1}{\left(i n_{A}-j n\right)} \cdot \frac{\partial H_{A}}{\partial l_{A}}\right)\right\}=0 .
$$

Now we are considering here the case in which the critical argument is at the point $n_{A}=2 n$ then the affected Hamiltonian is given by

$$
H_{A}=\frac{1}{2}\left(C_{4} X_{A} \cos l\right) \text {. }
$$

Taking $l=l_{A}-2(n t+\varepsilon)$ as the critical argument in our case so the Equation (53) becomes

$$
\frac{\mathrm{d}^{2} l}{\mathrm{~d} t^{2}}-\left(n_{A}-2 n\right)^{2} \frac{\mu_{A}}{2 K_{A}}\left[\frac{\partial}{\partial X_{A}}\left\{\frac{C_{4} X_{A}}{n_{A}-2 n}\right\}\right] \sin l=0 .
$$

As the first approximation, if we put $X_{A}=X_{A_{0}}, n_{A}=n_{A_{0}}, K_{A}=n_{A_{0}}$ (All constants) then Equation (54) becomes

$$
\frac{\mathrm{d}^{2} l}{\mathrm{~d} t^{2}}-\left(n_{A}-2 n\right)^{2} \frac{\mu_{A}}{2 K_{A_{0}}}\left[\frac{\partial}{\partial X_{A_{0}}}\left\{\frac{C_{4} X_{A}}{n_{A_{0}}-2 n}\right\}\right] \sin l=0 .
$$

This is the equation of motion of a simple pendulum. If co-efficient of $\sin l$ is negative then

$$
n_{\mathrm{A}}-2 n=0, \varepsilon_{\mathrm{A}_{0}}-2 \varepsilon=0 \text { or } \pi .
$$

If the oscillation is small, we can take $\sin l \approx l, n_{A}=n_{A_{0}}, X_{A}=X_{A_{0}}, K_{A}=K_{A_{0}}$ as $l$ oscillates about the value of 0 or $\pi$. Then Equation (56) becomes

$$
\begin{gathered}
\frac{\mathrm{d}^{2} l}{\mathrm{~d} t^{2}}-\Sigma\left(n_{A}-2 n\right)^{2} \frac{\mu_{A}}{2 K_{A_{0}}}\left[\frac{\partial}{\partial X_{A_{0}}}\left\{\frac{C_{4} X_{A}}{n_{A_{0}}-2 n}\right\}\right]_{0} l=0 \\
\frac{\mathrm{d}^{2} l}{\mathrm{~d} t^{2}}+P_{A}^{2} l=0
\end{gathered}
$$

where $P_{A}^{2}=\left|\frac{\mu_{A} C_{4}}{2 K_{A_{0}}} X_{A_{0}}\left(\frac{\partial n_{A}}{\partial X_{A_{0}}}\right)_{0}\right|$

$$
C_{4}=\left[\frac{I_{A_{3}} \omega_{A} \sin \theta_{A_{0}} 3 \mu\left(I_{A_{3}}-I_{A_{1}}\right)}{I_{A_{1}^{2}} 2 a^{3}\left(1-\mathrm{e}^{2}\right)^{3} m_{A}} \times \frac{\left(1+3 \mathrm{e}^{2}\right)}{2} \cdot \frac{1}{2 n}\right] .
$$


Its solution is given by

$$
l=Z_{A} \sin \left(P_{A} l+Z_{A_{0}}\right)
$$

where $Z_{A}$ and $Z_{A_{0}}$ are arbitrary constants. Thus amplitude and period of vibration are given by $Z_{A}$ and $\frac{2 \pi}{P_{A}}$ respectively with similar approximation in the first relation of Equation (50) and using the Equations (54) and (57), we get.

$$
X_{A}=X_{A_{0}}-\mu\left(\frac{C_{4} X_{A}}{2 K_{A}}\right) \frac{Z_{A}}{P_{A}} \cos \left(P_{A} l+Z_{A_{0}}\right)
$$

where $X_{A_{0}}$ can be determined from the equation $n_{A}=2 n$ as $n_{A_{0}}$ is known function.

\section{Equilibrium Points for the Body $A$ in Terms of Eulerian Angles}

Now we calculate the libration in the variables $\eta_{A}\left(\right.$ or $\left.=\psi_{A}\right)$ and $\varphi_{A}$.

Integrating the Equation (33) and ignoring secular terms, we get

$$
\begin{aligned}
\dot{\xi}_{A}= & \frac{3 \mu \mu_{A}}{2 a^{3}\left(1-\mathrm{e}^{2}\right)^{3} m_{A} I_{A_{1}}}\left(I_{A_{3}}-I_{A_{1}}\right) \sin 2 \theta_{A_{0}}\left(\frac{3 e}{2}-\frac{3 \mathrm{e}^{2}}{8}\right) \cdot \frac{1}{n} \sin +\frac{3 \mathrm{e}^{2}}{8} \sin 2(\theta-\omega) \\
& +\frac{\mathrm{e}^{3}}{24 n} \sin 3(\theta-\omega)+\frac{1}{4 n}\left(1+\frac{3 \mathrm{e}^{2}}{2}\right) \cdot \sin 2\left(\theta-\psi_{\mathrm{A}_{0}}\right) \\
& +\left(\frac{3 \mathrm{e}}{4}-\frac{3 \mathrm{e}^{3}}{10}\right) \cdot \frac{1}{3 n} \sin \left(3 \theta-2 \psi_{\mathrm{A}_{0}}-\omega\right)+\left(\frac{3 \mathrm{e}}{4}-\frac{3 \mathrm{e}^{3}}{16}\right) \cdot \frac{1}{n} \sin \left(\theta-2 \psi_{\mathrm{A}_{0}}+\omega\right) \\
& +\frac{3 \mathrm{e}^{2}}{32 n} \sin \left(4 \theta-2 \psi_{\mathrm{A}_{0}}-2 \omega\right)+\frac{\mathrm{e}^{3}}{80 n} \sin \left(5 \theta-2 \psi_{\mathrm{A}_{0}}-3 \omega\right) \\
& +\frac{\mathrm{e}^{3}}{16} \sin \left(\theta-3 \omega+2 \psi_{\mathrm{A}_{0}}\right)-\frac{I_{A_{3}}}{I_{A_{1}}} \omega_{A} \sin \theta_{\mathrm{A}_{0}} \eta_{A}
\end{aligned}
$$

where constants of integration are taken to be zero.

Putting the value of $\dot{\xi}_{A}$ in Equation (32) and ignoring secular term, we get

$$
\begin{aligned}
\ddot{\eta}_{A}-\left(\frac{\omega_{A} I_{A_{3}}}{I_{A_{1}}}\right)^{2} \eta_{A}= & \mu_{A}\left[c_{1} \sin (\theta-\omega)+c_{2} \sin 2(\theta-\omega)+c_{3} \sin (\theta-\omega)+c_{4} \sin 2\left(\theta-\psi_{A_{0}}\right)\right. \\
& +c_{5} \sin \left(3 \theta-2 \psi_{A_{0}}-\omega\right)+c_{6} \sin \left(\theta-2 \psi_{A_{0}}+\omega\right)+c_{7} \sin \left(4 \theta-2 \psi_{A_{0}}-2 \omega\right) \\
& \left.+c_{8} \sin \left(5 \theta-2 \psi_{A_{0}}-3 \omega\right)+c_{9} \sin \left(\theta+2 \psi_{A_{0}}-3 \omega\right)\right],
\end{aligned}
$$

where $c_{i}=1,2,3, \cdots, 9$ etc. are constants.

And the perturbed solution for $\eta_{A}$ is given by

$$
\begin{aligned}
\eta_{A}= & X_{A} \cos l_{A}+\mu_{A}\left[\frac{c_{1}}{-n^{2}+n_{A}^{2}} \sin (\theta-\omega)+\frac{c_{2}}{-4 n^{2}+n_{A}^{2}} \sin 2(\theta-\omega)+\frac{c_{3}}{-9 n^{2}+n_{A}^{2}} \sin (\theta-\omega)\right. \\
& +\frac{c_{4}}{-4 n^{2}+n_{A}^{2}} \sin 2\left(\theta-\psi_{A_{0}}\right)+\frac{c_{5}}{-9 n^{2}+n_{A}^{2}} \sin \left(3 \theta-2 \psi_{A_{0}}-\omega\right)+\frac{c_{6}}{-n^{2}+n_{A}^{2}} \sin \left(\theta-2 \psi_{A_{0}}+\omega\right) \\
& \left.\left.+\frac{c_{7}}{-16 n^{2}+n_{A}^{2}} \sin \left(4 \theta-2 \psi_{A_{0}}-2 \omega\right)+\frac{c_{8}}{-25 n^{2}+n_{A}^{2}} \sin \left(5 \theta-2 \psi_{A_{0}}-3 \omega\right)+\frac{c_{9}}{-n^{2}+n_{A}^{2}} \sin \left(\theta+2 \psi_{A_{0}}-3 \omega\right)\right]\right\}
\end{aligned}
$$


Obviously in the case of one of the denominator becomes zero, the motion cannot be determined at that point, known as critical point and hence resonance arise at that point. In this case usual method fails to determine the motion, so for the present purpose the present purpose we will use the method as that of $\xi_{A}$.

The equation for $\eta_{A}$ can be written as

$$
\begin{gathered}
\ddot{\eta}_{A}+n_{A}^{2} \eta_{A}=\mu_{A} \frac{\partial H_{A}^{\prime}}{\partial \eta_{A}} \\
H_{A}^{\prime}=\frac{X_{A}^{\prime}}{2}\left[c_{1}\left\{\sin \left(\theta-\omega+l_{A}\right)+\sin \left(\theta-\omega-l_{A}\right)\right\}\right. \\
+c_{2}\left\{\sin \left(2 \theta-2 \omega+l_{A}\right)+\sin \left(2 \theta-2 \omega-l_{A}\right)\right\} \\
+c_{3}\left\{\sin \left(3 \theta-3 \omega+l_{A}\right)+\sin \left(3 \theta-3 \omega-l_{A}\right)\right\} \\
+c_{4}\left\{\sin \left(2 \theta-2 \psi_{A_{0}}+l_{A}\right)+\sin \left(2 \theta-2 \psi_{A_{0}}-l_{A}\right)\right\} \\
+c_{5}\left\{\sin \left(3 \theta-2 \psi_{A_{0}}-\omega+l_{A}\right)+\sin \left(3 \theta-2 \psi_{A_{0}}-\omega-l_{A}\right)\right\} \\
+c_{6}\left\{\sin \left(\theta-2 \psi_{A_{0}}+\omega+l_{A}\right)+\sin \left(\theta-2 \psi_{A_{0}}+\omega-l_{A}\right)\right\} \\
+c_{7}\left\{\sin \left(4 \theta-2 \psi_{A_{0}}-2 \omega+l_{A}\right)+\sin \left(4 \theta-2 \psi_{A_{0}}-2 \omega-l_{A}\right)\right\} \\
+c_{8}\left\{\sin \left(5 \theta-2 \psi_{A_{0}}-3 \omega+l_{A}\right)+\sin \left(5 \theta-2 \psi_{A_{0}}-3 \omega-l_{A}\right)\right\} \\
\left.+c_{9}\left\{\sin \left(\theta+2 \psi_{A_{0}}-3 \omega+l_{A}\right)+\sin \left(\theta+2 \psi_{A_{0}}-3 \omega-l_{A}\right)\right\}\right] .
\end{gathered}
$$

On taking the first approximation, we can see that critical argument oscillates about $\frac{\pi}{2}$ or $\frac{3 \pi}{2}$. Also the solution for $l$ is given by

$$
l=Z_{A}^{\prime} \sin \left(P_{A}^{\prime} t+Z_{A_{0}}^{\prime}\right)
$$

where $Z_{A}^{\prime}$ and $Z_{A_{0}}^{\prime}$ are arbitrary constant.

Thus amplitude and period of vibration are given by $Z_{A}^{\prime}$ and $\frac{2 \pi}{P_{A}^{\prime}}$ respectively,

where $P_{A^{\prime}}^{2}=\left|\frac{\mu_{A} C_{4}}{2 K_{A_{0}}} X_{A_{0}^{\prime}}\left(\frac{\partial \eta_{A}}{\partial X_{A}^{\prime}}\right)\right|$,

$$
\begin{gathered}
K_{A}^{\prime}=\frac{\partial}{\partial X_{A}^{\prime}}\left(n_{A} \frac{\partial \eta_{A}}{\partial l_{A}}\right) \frac{\partial \eta_{A}}{\partial l_{A}}-n_{A} \frac{\partial^{2} \eta_{A}}{\partial l_{A}^{2}} \cdot \frac{\partial \eta_{A}}{\partial X_{A}} \\
c_{4}=\left\{\frac{3 \mu \omega_{A} I_{A_{3}}}{I_{A_{1}^{2}} 2 a^{3}\left(1-\mathrm{e}^{2}\right)^{3} m_{A}} \times\left(I_{A_{3}}-I_{A_{1}}\right) \times 2 \cos \theta_{A_{0}} \times\left(1+\frac{3 \mathrm{e}^{2}}{2}\right) \times \frac{1}{4 n}\right. \\
\left.+\frac{3 \mu\left(I_{A_{3}}-I_{A_{1}}\right)}{2\left(1-\mathrm{e}^{2}\right)^{3} a^{3} m_{A} I_{A_{1}}} \times\left(1+\frac{3 \mathrm{e}^{2}}{2}\right)\right\} .
\end{gathered}
$$

The solution for $X_{A}^{\prime}$ is given by

$$
X_{A}^{\prime}=X_{A_{0}}^{\prime}-\mu\left(\frac{c_{4} X_{A}^{\prime}}{2 K_{A}^{\prime}}\right)_{0} \times \frac{Z_{A}^{\prime}}{P_{A}^{\prime}}\left(P_{A}^{\prime} t+Z_{A_{0}}^{\prime}\right)
$$


where $X_{A}^{\prime}$ can be determined from the equation $n_{A_{0}}=2 n$ as $n_{A_{0}}$ is a known function. From the Equation (34) it is obvious that $\zeta_{A}$ depends on $\eta_{A}$ so that all the results of $\zeta_{A}$ can be found in terms of $\eta_{A}$.

\section{Equilibrium Points for the Body $B$ in Terms of Eulerian Angles}

By proceeding exactly same as above case, we can find out the libration in the variables $\theta_{B}, \psi_{B}$ and $\phi_{B}$. Here, we assume that $\left|2 n-n_{B}\right|$ is a small quantity and at the equilibrium point $2 n=n_{B}$ i.e. mean motion and angular velocity of the body $B$ are in the ratio of 1:2. Therefore at this point the resonance will arise. By taking

$$
\mu_{B}=1-\mu_{A}=\frac{m_{B}}{m_{B}+m_{A}}
$$

and the solution up to first order approximation of $\mu_{A}$, we get

$$
\begin{aligned}
& \xi_{B}=X_{B} \cos l_{B}+\mu_{B}\left[\frac{D_{1}}{-n^{2}+n_{B}^{2}} \cos (\theta-\omega)+\frac{D_{2}}{-4 n^{2}+n_{B}^{2}} \cos 2(\theta-\omega)\right. \\
& +\frac{D_{3}}{-9 n^{2}+n_{B}^{2}} \cos 3(\theta-\omega)+\frac{D_{4}}{-4 n^{2}+n_{B}^{2}} \cos 2\left(\theta-\psi_{B_{0}}\right) \\
& +\frac{D_{5}}{-9 n^{2}+n_{B}^{2}} \cos \left(3 \theta-2 \psi_{B_{0}}-\omega\right) \\
& +\frac{D_{6}}{-n^{2}+n_{B}^{2}} \cos \left(\theta-2 \psi_{B_{0}}+\omega\right) \\
& +\frac{D_{7}}{-16 n^{2}+n_{B}^{2}} \cos \left(4 \theta-2 \psi_{B_{0}}-2 \omega\right) \\
& +\frac{D_{8}}{-25 n^{2}+n_{B}^{2}} \cos \left(5 \theta-2 \psi_{B_{0}}-3 \omega\right) \\
& \left.+\frac{D_{9}}{-n^{2}+n_{B}^{2}} \cos \left(\theta+2 \psi_{B_{0}}-3 \omega\right)\right] \\
& \eta_{B}=X_{B}^{\prime} \cos l_{B}+\mu_{B}\left[\frac{d_{1}}{-n^{2}+n_{B}^{2}} \sin (\theta-\omega)+\frac{d_{2}}{-4 n^{2}+n_{B}^{2}} \sin 2(\theta-\omega)\right. \\
& +\frac{d_{3}}{-9 n^{2}+n_{B}^{2}} \sin 3(\theta-\omega)+\frac{d_{4}}{-4 n^{2}+n_{B}^{2}} \sin 2\left(\theta-\psi_{B_{0}}\right) \\
& +\frac{d_{5}}{-9 n^{2}+n_{B}^{2}} \sin \left(3 \theta-2 \psi_{B_{0}}-\omega\right) \\
& +\frac{d_{6}}{-n^{2}+n_{B}^{2}} \sin \left(\theta-2 \psi_{B_{0}}+\omega\right) \\
& +\frac{d_{7}}{-16 n^{2}+n_{B}^{2}} \sin \left(4 \theta-2 \psi_{B_{0}}-2 \omega\right) \\
& +\frac{d_{8}}{-25 n^{2}+n_{B}^{2}} \sin \left(5 \theta-2 \psi_{B_{0}}-3 \omega\right) \\
& \left.+\frac{d_{9}}{-n^{2}+n_{B}^{2}} \sin \left(\theta+2 \psi_{B_{0}}-3 \omega\right)\right]
\end{aligned}
$$

$X_{B}$ and $X_{B}^{\prime}$ are arbitrary constants. 


$$
l_{B}=n_{B} t+\epsilon_{B}, \quad n_{B}=\frac{\omega_{B} I_{B_{3}}}{I_{B_{1}}} .
$$

Also we see that in the libration in the variable $\theta_{B}$ the critical argument variable $l_{p}$ makes oscillation about the value 0 or $\pi$ and the period of libration is given by $\frac{2 \pi}{P_{B}}$.

The solution of $l_{p}$ for small oscillation is given by $l_{P}=Z_{B} \sin \left(P_{B} t+Z_{B_{0}}\right)$, where $Z_{B}$ and $Z_{B_{0}}$ are arbitrary constant.

$$
\begin{aligned}
P_{B}^{2}=[ & \frac{D_{4} X_{B}\left(1-\mu_{A}\right)}{2 K_{B}} \frac{\eta_{B}}{\partial X_{B}}+\frac{1-2 \mu_{A}}{K_{B}^{2}}\left\{\frac{\partial}{\partial X_{B}}\left(X_{B} D_{4}\right) \frac{D_{4} X_{B}}{K_{B}} \cdot \frac{\partial K_{B}}{\partial X_{B}}+\frac{\partial}{\partial X_{B}}\left(X_{B} D_{4}\right)\right\} \\
& \left.-D_{4} X_{B} \frac{\partial^{2}\left(X_{B} D_{4}\right)}{\partial X_{B}}\right]_{0}
\end{aligned}
$$

$K_{B}=\frac{\partial}{\partial X_{B}}\left(n_{B} \frac{\partial \xi_{B}}{\partial l_{A}}\right) \cdot \frac{\partial \xi_{B}}{\partial l_{B}}-n_{B} \frac{\partial^{2} \xi_{B}}{\partial X_{B}}$ is a function of $X_{B}$ only.

$$
\begin{aligned}
D_{4}= & {\left[\frac{-3 \mu\left(I_{B_{3}}-I_{B_{1}}\right)}{I_{B_{1}} 2 a^{\prime 2}\left(1-\mathrm{e}^{2}\right)^{3} m_{B}} \sin ^{2} \theta_{B_{0}}\left(\frac{1}{2}+\frac{3 \mathrm{e}^{2}}{4}\right)+\frac{-3 \mu I_{B_{3}} \omega_{B} \sin \theta_{B_{0}}\left(I_{B_{3}}-I_{B_{1}}\right)}{I_{B_{1}}^{2} 2 a^{\prime 3}\left(1-\mathrm{e}^{2}\right)^{3} m_{B}}\right.} \\
& \left.\times\left(1+\frac{3 \mathrm{e}^{2}}{2}\right) \frac{1}{2 n}\right] .
\end{aligned}
$$

Solution for $X_{B}$ is given by

$$
X_{B}=X_{B_{0}}-\frac{\mu_{B}}{2}\left(\frac{D_{4} X_{B}}{K_{B}}\right)_{0} \frac{Z_{B}}{P_{B}} \cos \left(P_{B} t+Z_{B_{0}}\right) .
$$

Also when we consider the libration in the variable $\psi_{B}$ we see that the critical argument $l_{P}$ will make oscillation about the value $\frac{\pi}{2}$ or $\frac{3 \pi}{2}$ and the period of libration is given by $\frac{2 \pi}{P_{B}}$.

The solution of $l_{P}$ for small oscillation in this case will be $l_{P}=Z_{B}^{\prime} \sin \left(P_{B}^{\prime} t+Z_{B_{0}}^{\prime}\right)$, where $Z_{B}^{\prime}$ and $Z_{B_{0}}^{\prime}$ are arbitrary constant.

$$
\begin{gathered}
P_{B^{\prime}}^{2}=\left[\frac{d_{4} X_{B^{\prime}}^{\prime}\left(1-\mu_{A}\right)}{2 K_{B}^{\prime}} \frac{\partial \eta_{B}}{\partial X_{B}}+\frac{1-2 \mu_{A}}{K_{B}^{2}}\left\{\frac{\partial}{\partial X_{B}^{\prime}}\left(X_{B}^{\prime} d_{4}\right)\left(\frac{d_{4} X_{B}^{\prime}}{K_{B}^{\prime}} \cdot \frac{\partial K_{B}^{\prime}}{\partial X_{B}}+\frac{\partial}{\partial X_{B}^{\prime}}\left(d_{4} X_{B}\right)\right)\right.\right. \\
\left.\left.-d_{4} X_{B}^{\prime} \frac{\partial^{2}}{\partial X_{B^{\prime}}^{2}}\left(X_{B}^{\prime} d_{4}\right)\right\}\right]_{0} \\
K_{B}^{\prime}=\frac{\partial}{\partial X_{B}^{\prime}}\left(n_{B} \frac{\partial \eta_{B}}{\partial l_{B}}\right) \cdot \frac{\partial \eta_{B}}{\partial l_{B}}-n_{B} \frac{\partial^{2} \eta_{B}}{\partial l_{B}^{2}} \cdot \frac{\partial \eta_{B}}{\partial X_{B}} \\
d_{4}=\left[\frac{3 \mu \omega_{B} I_{B_{3}} \cdot\left(I_{B_{3}}-I_{B_{1}}\right)}{I_{B_{3}}^{2} 2 a^{\prime 3}\left(1-\mathrm{e}^{2}\right)^{3} m_{A}} 2 \cos \theta_{B_{0}}\left(\frac{3 \mathrm{e}}{4}-\frac{3 \mathrm{e}^{3}}{16}\right) \cdot \frac{1}{4 n}+\frac{3 \mu\left(I_{B_{3}}-I_{B_{1}}\right)}{2\left(1-\mathrm{e}^{2}\right)^{3} a^{\prime 3} m_{A} I_{B_{1}}}\left(1+\frac{3 \mathrm{e}^{3}}{2}\right)\right] .
\end{gathered}
$$

And the solution for $X_{B}^{\prime}$ is given by 


$$
X_{B}^{\prime}=X_{B_{0}}^{\prime}-\frac{\mu_{B}}{2}\left(\frac{d_{4} X_{B}^{\prime}}{K_{B}^{\prime}}\right)_{0} \frac{Z_{B}^{\prime}}{P_{B}^{\prime}} \cos \left(P_{B}^{\prime} l+Z_{B_{0}}^{\prime}\right) .
$$

where $X_{B}^{\prime}$ can be determined from the equation $n_{B_{0}}=2 n$ as $n_{B_{0}}$ is a known function.

From the Equation (37) it is obvious and $\zeta_{B}$ depends on $\eta_{B}$, so that the result of $\zeta_{B}$ can be found in term of $\eta_{B}$.

\section{The Solution for the Generalized Momenta Variables Corresponding to Constants of Integration}

We have from Equation (16),

$$
a=\text { constant }=a_{0}, e=\text { constant }=e_{0} .
$$

Integrating the Equation (17) with respect to $t$, we get

$$
\begin{gathered}
\beta_{1}=\frac{F t}{\left(1-\mathrm{e}^{2}\right)^{3 / 2}}+\text { constant } \\
F=\frac{3}{a^{2}\left(1-\mathrm{e}^{2}\right)^{3 / 2}}\left[-\frac{\left(I_{A_{3}}-I_{A_{1}}\right)}{2 m_{A}}\left(1-\frac{3}{2} \sin ^{2} \theta_{A}\right)-\frac{\left(I_{B_{3}}-I_{B_{1}}\right)}{2 m_{B}}\left(1-\frac{3}{2} \sin ^{2} \theta_{B}\right)\right] .
\end{gathered}
$$

Initially at $t=0$ take $\beta_{1}=\tau_{0}$ and using the Equation (16), we get

$$
\tau=M t+\tau_{0}
$$

where, $M=\frac{-F}{\left(1-\mathrm{e}_{0}^{2}\right)^{3 / 2}}$.

Again from Equation (18), we have

$$
\beta_{2}=\frac{n F t}{\left(1-\mathrm{e}_{0}^{2}\right)^{3 / 2}}+\text { constant. }
$$

Initially at $t=0$ take $\beta_{2}=\omega_{0}$ and using the Equation (14), we get

$$
\beta_{2}=\omega=\frac{-F t}{\left(1-\mathrm{e}_{0}^{2}\right)^{2}}+\omega_{0}=N n t+\omega_{0},
$$

where, $\quad N=\frac{-F}{\left(1-\mathrm{e}_{0}^{2}\right)^{2}}$.

Now we find the time $\Delta t$ that elapses between the instant at which $r$ attains successive minima and $\Delta \theta$ the corresponding change in $\theta$.

We have $r=a(1-e \cos E)=a_{0}\left(1-e_{0} \cos E\right)$. Clearly $r$ attains it successive minima at $E=0$ or $E=2 \pi$.

Let $E=0$ when $t=t_{1}$ and $E=2 \pi$ when $t=t_{2}$. Then from Equations (14) and (34), we have

$$
\Delta t \cong \frac{2 \pi}{n(1-M)} .
$$

Again from the Equations (13) and (36), we get 


$$
\theta=N n t+\omega_{0}+\cos ^{-1}\left[\frac{(\cos E-e)}{1-e \cos E}\right]
$$

Let $E=0$ when $\theta=\theta_{1}$ and $E=2 \pi$ when $\theta=\theta_{2}$.

The corresponding change in $\theta$ is given by $\Delta \theta=2 \pi\left[1+\frac{N}{1-M}\right]$.

\section{Conclusions}

In the section of "Equations of motion", we have derived the perturbed and unperturbed Hamiltonian and the canonical equations of motion with respect to the complete Hamiltonian $\mathrm{H}$ where are generalized co-ordinates and are the corresponding generalized momenta. In Section 3, unperturbed solutions can be derived by usual course from the Kepler's equation of motion. For appropriate variational equation, the required generalized co-ordinates have been calculated in Section 5. In section 6, the effect of resonance has been shown in the solutions of the equations of motion of two cylindrical rigid bodies. In Section 7 and 8 , equilibrium points have been calculated in terms of Eulerian angles for both the bodies.. Finally the appropriate variational equation in Section 4 has been completely solved in Section 9.

The tools obtained in different sections of the manuscript can be used to discuss the motion of cable connected two artificial satellites. Thus, we may conclude that this article is highly applicable in Astrophysics and Space Science.

\section{References}

[1] Bhatnagar, K.B. and Gupta, B. (1977) Resonance in the Restricted Problems of Three Bodies with Short Periodic Perturbations. Proceedings of the Indian National Science Academy, Vol. 43, 153-168.

[2] Bhatnagar, K.B. and Gupta, B. (1977) Resonance in the Restricted Problems of Three Bodies with Short Periodic Perturbations in the Elliptic Case. Proceedings of the Indian National Science Academy, Vol. 43, 290-302.

[3] Bhatnagar, K.B. (1978) Motion of Two Rigid Bodies under the Gravitational Influence of Each Other. Astronomy and Astrophysics, 62, 217-221.

[4] Russel, H.N. (1928) On the Advance of Periastron in Eclipsing Binaries. Monthly Notices of Royal Astronomical Society, 88, 641-643. https://doi.org/10.1093/mnras/88.8.641

[5] Kopal, Z. (1938) On the Motion of the Apsidal Line in Close Binary Systems. Monthly Notices of Royal Astronomical Society, 98, 448-458. https://doi.org/10.1093/mnras/98.6.448

[6] Cowling, T.G. (1938) On the Motion of Apsidal Line in Close Binary Systems. Monthly Notices of Royal Astronomical Society, 98, 734-743. https://doi.org/10.1093/mnras/98.9.734

[7] Sterne, T.E. (1938) Apsidal Motion in Binary Stars (II) Distributions of Density. Monthly Notices of Royal Astronomical Society, 99, 662-670. https://doi.org/10.1093/mnras/99.8.662

[8] Brouwer, D. (1946) A Survey of the Dynamics of Close Binary Systems. The Astronomical Journal, 52, 57-62. https://doi.org/10.1086/105913

[9] Johnson, D.B. and Kane, T.R. (1969) On a Restricted Problem of Two Rigid Spheroids. The 
Astronomical Journal, 14, 563-567. https://doi.org/10.1086/110835

[10] Antonio, E. and Vallejo, M. (2001) On the Attitude Dynamics of Perturbed Triaxial Bodies. Celestial Mechanics and Dynamical Astronomy, 81, 1-2. https://doi.org/10.1023/A:1017437130947

[11] Choudhary, R.K. and Mishra, P.K. (1974) Restricted Problem of a Rigid Spheroid and an Ellipsoid. Journal of Indian Mathematical Society, 38, 305-317.

[12] Mercedes, A. and Elipe, A. (1993) Attitude Dynamics of a Rigid Body on a Keplerian Orbit-A Simplification. Celestial Mechanics and Dynamical Astronomy, 55, 243-247. https://doi.org/10.1007/BF00692512

[13] Marjanov, M. (2007) Two Real Bodies Problem-Complex Harmony of Motions. Mechanics, Automatic Control and Robotics, 6, 65-73.

[14] Brouwer, D. and Clemence, M. (1971) Methods of Celestial Mechanics. Academic Press, New York, London.

[15] Brown, E.N. and Shook, C.A. (1933) Planetary Theory. Cambridge University Press, Cambridge, United Kingdom.

Submit or recommend next manuscript to SCIRP and we will provide best service for you:

Accepting pre-submission inquiries through Email, Facebook, LinkedIn, Twitter, etc. A wide selection of journals (inclusive of 9 subjects, more than 200 journals)

Providing 24-hour high-quality service

User-friendly online submission system

Fair and swift peer-review system

Efficient typesetting and proofreading procedure

Display of the result of downloads and visits, as well as the number of cited articles Maximum dissemination of your research work

Submit your manuscript at: http://papersubmission.scirp.org/

Or contact ijaa@scirp.org 